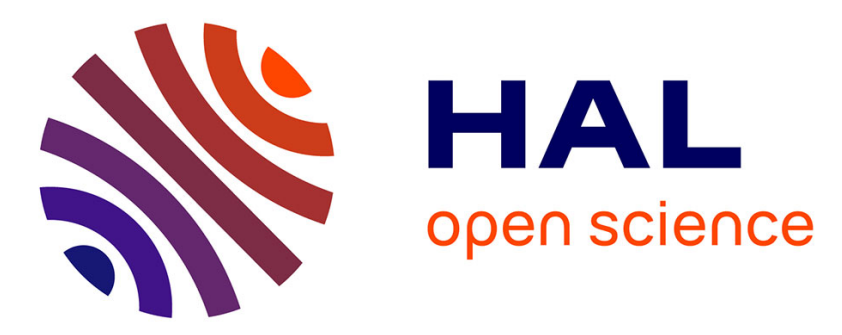

\title{
Mannosylation Of Budesonide Palmitate Nanoprodrugs For Improved Macrophage Targeting
}

Ludmila Pinheiro Do Nascimento, Nicolas Tsapis, Franceline Reynaud, Didier

Desmaële, Laurence Moine, Juliette Vergnaud, Sonia Abreu, Pierre

Chaminade, Elias Fattal

\section{To cite this version:}

Ludmila Pinheiro Do Nascimento, Nicolas Tsapis, Franceline Reynaud, Didier Desmaële, Laurence Moine, et al.. Mannosylation Of Budesonide Palmitate Nanoprodrugs For Improved Macrophage Targeting. European Journal of Pharmaceutics and Biopharmaceutics, 2021, 170, pp.112-120. 10.1016/j.ejpb.2021.12.001 . hal-03475936

\section{HAL Id: hal-03475936 https://hal.science/hal-03475936}

Submitted on 11 Dec 2021

HAL is a multi-disciplinary open access archive for the deposit and dissemination of scientific research documents, whether they are published or not. The documents may come from teaching and research institutions in France or abroad, or from public or private research centers.
L'archive ouverte pluridisciplinaire HAL, est destinée au dépôt et à la diffusion de documents scientifiques de niveau recherche, publiés ou non, émanant des établissements d'enseignement et de recherche français ou étrangers, des laboratoires publics ou privés. 


\section{MANNOSYLATION OF BUDESONIDE PALMITATE NANOPRODRUGS FOR IMPROVED MACROPHAGE TARGETING}

Ludmila Pinheiro do Nascimento ${ }^{1}$, Nicolas Tsapis ${ }^{1}$, Franceline Reynaud ${ }^{1,2}$, Didier Desmaële $^{1}$, Laurence Moine ${ }^{1}$, Juliette Vergnaud ${ }^{1}$, Sonia Abreu $^{3}$, Pierre Chaminade ${ }^{3}$, Elias Fattal $^{1 *}$

${ }^{1}$ Université Paris-Saclay, CNRS, Institut Galien Paris-Saclay, 92296, Châtenay-Malabry, France.

${ }^{2}$ School of Pharmacy, Federal University of Rio de Janeiro, 21944-59 Rio de Janeiro, Brazil

${ }^{3}$ Université Paris-Saclay, Lipides, Systèmes Analytiques et Biologiques, 92296, ChâtenayMalabry, France.

*Corresponding author :

Elias Fattal

Institut Galien Paris-Saclay

UMR CNRS 8612

School of Pharmacy

Université Paris-Saclay

5 , rue J.B. Clément

92296 Châtenay-Malabry

France 


\begin{abstract}
In a strategy to improve macrophage targeting of glucocorticoids (GCS) for anti-inflammatory therapy, a so-called nanoprodrug of budesonide palmitate decorated by mannose moieties was designed. The synthesis of budesonide palmitate (BP) was obtained by esterification and mannosylated lipid (DSPE-PEG-Man) by reacting 1,2-Distearoyl-sn-Glycero-3-Phosphoethanolamine (DSPE)-polyethylene glycol-amine and $\alpha$-D-mannopyranosylphenyl isothiocyanate (MPITC). Nanoparticles were formulated by emulsion-evaporation and different ratios of mannosylated lipid were introduced in the formulation of BP nanoprodrugs. Using up to 75\% of DSPE-PEG-man (75/25) led to $200 \mathrm{~nm}$ particles with a polydispersity index below 0.2 , a negative zeta potential ranging from 10 to $-30 \mathrm{mV}$, and one-month stability at $4^{\circ} \mathrm{C}$. The encapsulation efficiency of BP approached $100 \%$ proving that the prodrug was associated with the particles, leading to a final BP loading of 50 -to $60 \%$ $(w / w)$. The lectin agglutination test confirmed the availability of mannose on the nanoprodrug surface. Nanoprodrug uptake by RAW 264.7 macrophages was observed by confocal microscopy and flow cytometry. After 24 and 48 hours of incubation, a significantly greater internalization of mannosylated nanoparticles as compared to PEGylated nanoparticles was achieved. The mannose receptor-mediated uptake was confirmed by a mannan inhibition study. After LPS-induced inflammation, the anti-inflammatory effect of mannosylated nanoparticles was assessed. After 48 hours of incubation, cytokines (MCP-1 and TNF $\alpha$ ) were reduced demonstrating that the functionalization of nanoprodrugs is possible and efficient.
\end{abstract}

Keywords: Nanoprodrugs, macrophages, targeting, mannose receptor 

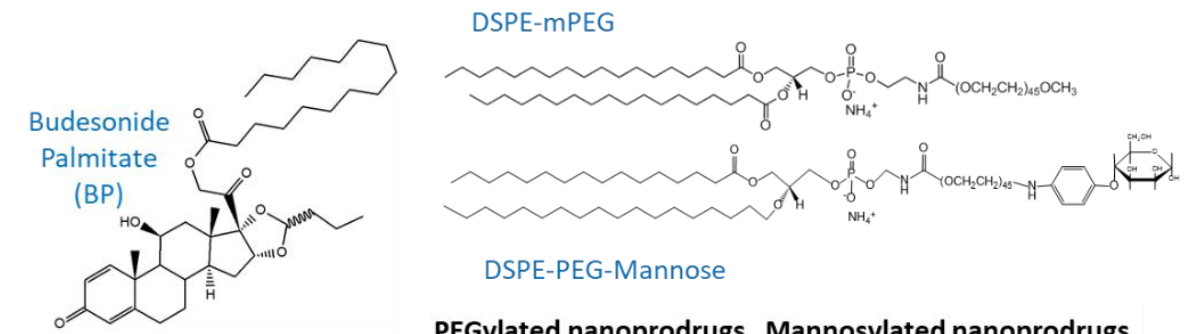

Anti-inflammatory effect

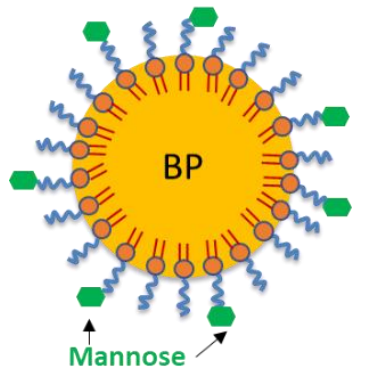

DSPE-PEG-Mannose

PEGylated nanoprodrugs Mannosylated nanoprodrugs
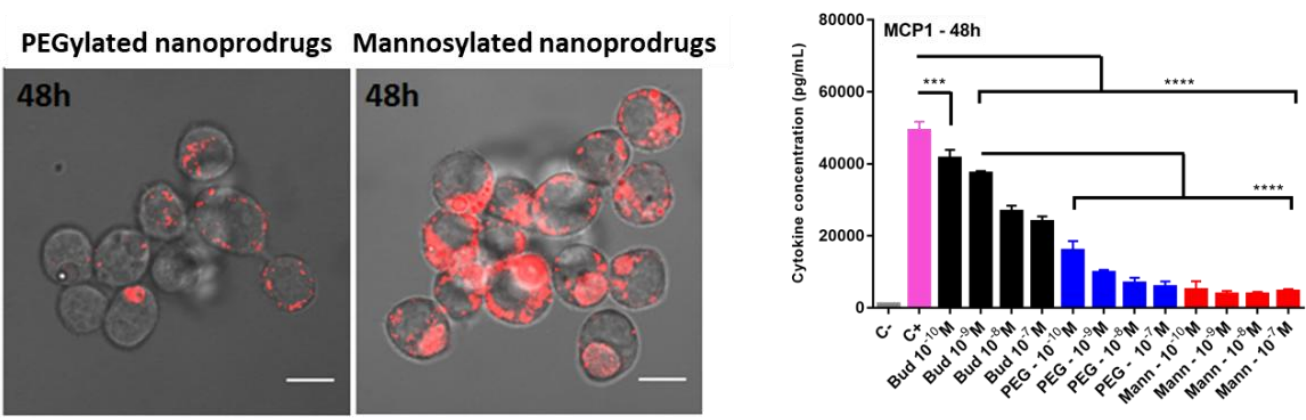

Increased macrophage uptake 


\section{Introduction}

Glucocorticoids (GCS) have been extensively applied in clinics as anti-inflammatory and immunosuppressive drugs despite their strong side effects due to unfavorable pharmacokinetic and biodistribution properties [1,2]. Indeed, administration of GCs is often associated with the risk of adverse events, especially when given at high doses and for a long time[3]. The most critical are diabetes, high blood pressure, or Cushing syndrome [1,2]. To improve GCs pharmacokinetics and increase their concentrations in inflamed therapeutic sites, the use of nanoparticles coated with poly(ethylene glycol) has been considered due to their long-circulating properties in the bloodstream [4] and their passive diffusion through highly permeable vasculature of inflamed tissues [5]. To overcome the low drug loading, the difficulty to entrap hydrophobic drugs $[6,7]$, and the premature burst release $[6,8,9]$ that severely hinders their clinical translation, we have recently designed a new process that was applied to the entrapment of GCs consisting of the association of a prodrug of dexamethasone, dexamethasone palmitate into a nanoparticle, called nanoprodrug, stabilized by the sole presence of Distearoyl-sn-Glycero-3-Phosphoethanolamine- $\mathrm{N}$-[methoxy (polyethylene glycol)2000)] (DSPE-mPEG) $[10,11]$. This novel type of drug carrier combines the advantages of prodrugs and nanoparticles making them very different from other lipid-based nanoparticles such as liposomes. They are easy to produce with a high loading efficiency and leading to modified pharmacokinetics and tissue distribution after IV administration due to a slow drug release process by ester bond hydrolysis [11]. In a collagen-induced arthritis model, these nanoprodrugs led to specific joint accumulation, a strong reduction of joint inflammation and histopathological signs of arthritis without increased side effects [10].

To better target GCs to macrophages, key cells in the inflammatory process, the nanoprodrugs could be functionalized with a specific ligand. Our group has recently shown that decorating polymeric nanoparticles with mannose on their surface led to increased uptake by alveolar macrophages after intratracheal instillation in mice [12]. Because of the presence of mannose receptors overexpressed on the surface of macrophages, that can recognize mannose with high affinity, the development of macrophages targeting systems using different carriers could improve the therapeutic efficiency of GCs and minimize drug side effects [13]. While mannose was coupled to different carriers made of polymers [14] and liposomes [15], it was never associated with lipid prodrugs despite the large interest in using and combining both strategies. Here, a lipophilic derivative of budesonide, budesonide palmitate (BP) was synthesized as a candidate for lung delivery to macrophages. it was formulated into a nanoprodrug by associating to the prodrug, DSPE-PEG with a methoxy end group (DSPE-mPEG) and a mannosylated lipid, DSPE-PEG-Man. The formulation process was optimized to ensure enough mannose on the nanoprodrug surface. Nanoprodrugs were characterized and their 
ability to target macrophages and reduce inflammation was evaluated on RAW 264.7 macrophages activated by lipopolysaccharide (LPS).

\section{Materials and methods}

\subsection{Materials}

Budesonide (Bud) was obtained from Chemos GmbH \& Co (Germany). DSPE-PEG-NH $\mathrm{N}_{2}$ (1,2-Distearoylsn-Glycero-3-Phosphoethanolamine-N-(methoxy(polyethylene glycol)-2000) (ammonium salt), DSPEmPEG (1,2-Distearoyl-sn-Glycero-3-Phosphoethanolamine-N-(amino(polyethylene glycol)-2000) (ammonium salt) and lissamine-rhodamine B sulfonyl were purchased from Avanti Polar Lipids, Inc. (USA). Palmitoyl chloride, pyridine, triethylamine, ammonium acetate, $\alpha$-D-Mannopyranosylphenyl isothiocyanate (MPITC), Methyl $\alpha$-D-mannopyranoside, Concanavalin A type IV, Mannan (isolated from Saccharomyces cerevisiae), and LPS (Lipopolysaccharides from Escherichia coli 055:B5) were provided by Sigma-Aldrich. Dialysis membrane Spectra/Por 6 Dialysis Tubing 1kD MWCO was purchased from Spectrum labs. Chloroform HPLC-grade, methanol HPLC-grade, acetone, and DMSO were purchased from Carlo Erba Reagents (France). All other chemicals were obtained commercially and were of the highest available analytical grade. Water was purified using a Milli Q Reference system (Merck Millipore, France).

\subsection{Synthesis of Budesonide Palmitate}

Budesonide palmitate, a prodrug of budesonide, was synthesized from the esterification of the alcohol-terminus present on the C-21 carbon of budesonide by the addition of palmitoyl chloride (Figure 1). Briefly, $1 \mathrm{mmol}$ of budesonide was dissolved in pyridine $(10.8 \mathrm{~mL}$ for each $\mathrm{mmol}$ of budesonide) and 2 equivalents of palmitoyl chloride were injected dropwise at $0^{\circ} \mathrm{C}$. The mixture remained at room temperature overnight under stirring and nitrogen. After 24 hours of reaction, the pyridine was distilled off.

The obtained solution was resuspended in dichloromethane (at least $10 \mathrm{~mL}$ ) and washed successively with approximately $5 \mathrm{~mL}$ of $\mathrm{HCl}(0.05 \mathrm{M}), 5 \mathrm{~mL}$ of distilled water, and $5 \mathrm{~mL}$ of $5 \% \mathrm{NaHCO}_{3}$ to neutralize the acid. The organic phase was dried using anhydrous magnesium sulfate $\left(\mathrm{MgSO}_{4}\right)$ for 30 minutes under stirring, and, after evaporation, the obtained solid was purified by silica gel column chromatography with ethyl acetate/cyclohexane $1 / 4$ (v/v)25. The separation was performed by thinlayer chromatography with the same eluent as mentioned above.

The fractions containing the product were collected in a flask, and the solvent was evaporated using a rotary evaporator and a $40^{\circ} \mathrm{C}$ bath. After weighing, the obtained products were resuspended in 
dichloromethane and distributed in small amber vials, remaining in a laminar flow hood for 3 hours until total evaporation of the solvent. The obtained ester was analyzed by nuclear magnetic resonance $\left({ }^{1} \mathrm{H} N M R\right)$ at $300 \mathrm{MHz}$ in deuterated chloroform $\left(\mathrm{CDCl}_{3}\right)$, with the following results: the presence of two isomers induced the splitting of most peaks $\delta 7.25(\mathrm{~d}, J=10.0 \mathrm{~Hz}, 1 \mathrm{H}, \mathrm{H}-1), 6.26(\mathrm{~d}, J$ $=10.0 \mathrm{~Hz}, 1 \mathrm{H}, \mathrm{H}-2), 6.00(\mathrm{~s}, 1 \mathrm{H}, \mathrm{H}-4), 5.17-5.09(\mathrm{~m}, 1 \mathrm{H}, \mathrm{OCHO}), 4.92-4.70(\mathrm{~m}, 2.4 \mathrm{H}, 2 \mathrm{H}-21,0.4 \mathrm{H}-$ 16), $4.60(\mathrm{t}, J=4.5 \mathrm{~Hz}, 0.6 \mathrm{H}, \mathrm{H}-16$ ), 4.48 (broad s, $1 \mathrm{H}, \mathrm{H}-11$ ), $2.52(\mathrm{td}, J=14.5 \mathrm{~Hz}, J=4.8 \mathrm{~Hz}, 1 \mathrm{H}, \mathrm{H}-6 \beta$ ), $2.36\left(\mathrm{t}, J=7.6 \mathrm{~Hz}, 2 \mathrm{H}, \mathrm{O}_{2} \mathrm{CH}_{2} \mathrm{CH}_{2}\right), 2.36-2.28(\mathrm{~m}, 1 \mathrm{H}, \mathrm{H}-6 \alpha), 2.2-1.5(\mathrm{~m}, 14 \mathrm{H}), 1.43$ and $1.42(\mathrm{~s}, 3 \mathrm{H}, \mathrm{H}-$ 19), 1.45-1.05 (m, 24H,), 1.00-0.83 (m, 9H, H-20, OCH(O) $\mathrm{CH}_{2} \mathrm{CH}_{2} \mathrm{CH}_{3}$, palmCH$)$.

\subsection{Synthesis of DSPE-PEG-Mannose}

The synthesis of DSPE-PEG-Mannose (DSPE-PEG-Man) was performed by a chemical reaction between DSPE-PEG-NH ${ }_{2}$ and MPITC [16-18]. DSPE-PEG-NH $\left(\mathrm{Mw}=2790.486 \mathrm{~g}^{-\mathrm{mol}^{-1}}\right)$ and MPITC (Mw $=313.33 \mathrm{~g} \cdot \mathrm{mol}^{-1}$ ) were dissolved in DMSO at a 1:3 ratio, with the reaction being kept under stirring at $25^{\circ} \mathrm{C}$ for $16 \mathrm{~h}$. The product obtained, DSPE-PEG-Man, was then mixed with Milli Q water (1:10) and dialyzed for $48 \mathrm{~h}$ to remove free mannose (distilled water, $1 \mathrm{kDa}$ membrane) and lyophilized at the end. The chemical structure of DSPE-PEG-Man was characterized by ${ }^{1} \mathrm{H}$ NMR (Figure 1).

\subsection{Nanoprodrug formulation}

Nanoprodrugs were formulated using emulsion-evaporation method to obtain 5 samples with final BP concentration $1.25 \mathrm{mg} / \mathrm{mL}$ and different DSPE-PEG-Man/DSPE-mPEG ratios to a final lipid concentration $1.25 \mathrm{mg} / \mathrm{mL}$ : 100/0,75/25, 50/50, 25/75, and 0/100, respectively (Table 1 ), using a protocol recently published [10].

Briefly, nanoprodrugs were prepared by solubilizing the lipids DSPE-PEG-Man/DSPE-mPEG and BP in $1 \mathrm{~mL}$ of chloroform. This organic phase was slowly injected with a 20G needle (Braun, Sterican, 0.90 by $70 \mathrm{~mm}$ and $20 \mathrm{G} \times 23 / 4$ ") into an aqueous phase composed of 5 or $10 \mathrm{~mL}$ of Milli $\mathrm{Q}$ water at $4^{\circ} \mathrm{C}$, with subsequent vortexing. The emulsion formed was then sonicated (Branson Digital Sonifier) for 2 minutes at an amplitude of $40 \%(300 \mathrm{~W})$. The solvent was then evaporated under reduced pressure using a rotary evaporator (Buchi, R-124). The final volume was adjusted, when necessary, in a volumetric flask to 5 or $10 \mathrm{~mL}$, according to the defined concentration. 
Table 1. Mannosylated (M) and PEGylated nanoprodrugs composition

\begin{tabular}{|c|c|c|}
\hline $\begin{array}{c}\text { DSPE-PEG-Man } \\
\text { /DSPE-mPEG } \\
\text { ratio }\end{array}$ & $\begin{array}{c}\text { Conc. DSPE- } \\
\text { PEG-Man } \\
(\mathrm{mg} / \mathrm{mL})\end{array}$ & $\begin{array}{c}\text { Conc. DSPE- } \\
\mathrm{mPEG} \\
(\mathrm{mg} / \mathrm{mL})\end{array}$ \\
\hline $100 / 0$ & 1.25 & 0 \\
\hline $75 / 25$ & 0.937 & 0.313 \\
\hline $50 / 50$ & 0.625 & 0.625 \\
\hline $25 / 75$ & 0.313 & 0.937 \\
\hline
\end{tabular}

\subsection{Nanoprodrug size and zeta potential}

Nanoprodrugs were characterized by their size, polydispersity index (Pdl), and zeta potential, measured by dynamic light scattering with a Nano ZS (Malvern Instruments, UK) at a $173^{\circ}$ scattering angle at $25^{\circ} \mathrm{C}$. Samples were diluted $1 / 20$ in water for the size and $\mathrm{Pdl}$, or in $1 \mathrm{mM} \mathrm{NaCl}$ solution for the zeta potential determination. Measurements were performed in triplicate.

\subsection{Transmission Electron Microscopy}

Transmission electron microscopy was performed at I2BC (CNRS, Gif-sur-Yvette, France). A volume of $5 \mu \mathrm{L}$ of the nanoparticle suspension at $5 \mathrm{mg} / \mathrm{mL}$ BP was deposited for 1 minute on 400 mesh formwar-coated copper grids. Negative staining was performed by addition of a drop of uranyl acetate at $2 \% \mathrm{w} / \mathrm{w}$ for 30 seconds. Excess solution was removed and grids were left to dry before observation. The observations were carried out on a JEOL JEM-1400 microscope at an acceleration voltage of $80 \mathrm{kV}$. Images were acquired using an Orius camera (Gatan Inc, USA).

\subsection{Drug encapsulation efficiency and Loading}

To evaluate the encapsulation efficiency and the prodrug loading, nanoprodrug suspensions were treated with methanol to extract the prodrug and lipids and further quantify the total BP and DSPEPEG-Man by HPLC. To separate the free compounds from the nanoprodrugs, ultracentrifugation was performed at $40,000 \mathrm{rpm}\left(=109760 \mathrm{~g}\right.$ ) for $4 \mathrm{~h}$ at $4^{\circ} \mathrm{C}$ (Beckman Coulter Optima LE-80K ultracentrifuge, 70-1Ti rotor). After separation by ultracentrifugation, non-encapsulated BP and DSPE-PEG-Man in the supernatant were quantified by HPLC-UV and their amount in nanoprodrugs was calculated indirectly. Analyzes were performed using an Agilent chromatograph, equipped with a UV detector (785A, Applied Biosystems) coupled to an evaporative light-scattering detector (ELSD, Eurosep, Cergy, France). The UV detection was performed at $244 \mathrm{~nm}$ wavelength and ELSD detection settings were a nebulization temperature of $35^{\circ} \mathrm{C}$ and an evaporation temperature of $45^{\circ} \mathrm{C}$. The Waters SymmetryShield RP18 column with $5 \mu \mathrm{m}$ particles and $4.6 \times 250 \mathrm{~mm}$ dimensions was maintained at 
$10^{\circ} \mathrm{C}$ with a cooler. The isocratic mobile phase was composed of methanol: Chloroform: Ammonium acetate $\mathrm{pH} 4.0(95: 3: 2, \mathrm{v}: \mathrm{v}: \mathrm{v})$, with the addition of triethylamine $25 \mu \mathrm{L} \%+$ acetic acid $30 \mu \mathrm{L} \%$. The flow rate was $1.0 \mathrm{~mL} / \mathrm{min}$. A volume of $30 \mu \mathrm{L}$ of samples was injected and analyzed during $12 \mathrm{~min}$. UV Retention times were $6.5 \mathrm{~min}$ and $9.7 \mathrm{~min}$ for DSPE-PEG-Man and BP, respectively. The concentration ranges of the calibration curves were $0.1-3 \mathrm{mg} / \mathrm{mL}$ for DSPE-PEG-Man and $10-750 \mu \mathrm{g} / \mathrm{mL}$ for BP. UV calibration curve followed a linear model, $y=2185 x-42.883, R^{2}=0.9838$ (DSPE-PEG-Man, LOQ=690ng, $L O D=230 n g$ ) and $y=27.359 x+54.682, R^{2}=0.9994$ ( $B P, L O Q=60 n$, LOD=20ng). ELSD Retention times were $6 \mathrm{~min}$ and $6.5 \mathrm{~min}$ for DSPE-mPEG and DSPE-PEG-NH , respectively. The concentration ranges of the calibration curves were $18.7-750 \mu \mathrm{g} / \mathrm{mL}$ for DSPE-mPEG and $18.7-500 \mu \mathrm{g} / \mathrm{mL}$ for DSPE-PEG-NH ${ }_{2}$. ELSD detection calibration curves followed a power law model, equations and correlation coefficients were $y=0.0039 x^{1.4907}, R^{2}=0.9959$ for DSPE-mPEG (LOQ=150ng, LOD=50ng) and $y=0.6532 x^{1.4494}$, $\mathrm{R}^{2}=0.998$ for DSPE-PEG-NH $(\mathrm{LOQ}=93 \mathrm{ng}, \mathrm{LOD}=31 \mathrm{ng}$ ). The encapsulation efficiency was calculated as a percentage of the ratio of the actual amount of the compound over the initial one. The prodrug loading was determined as a weight percentage of the weight of prodrug encapsulated over the total weight of nanoparticle compounds (Prodrug + DSPE-PEG-Man + DSPE-mPEG) added to the formulation. Similar calculations were performed for DSPE-PEG-Man.

\subsection{Lectin Agglutination Test}

The presence of mannose particles on the surface of the nanoprodrugs was evaluated qualitatively by the agglutination assay with the concanavalin A (ConA) lectin. Lectins are proteins that specifically and reversibly interact with carbohydrates, forming precipitates. Among the commonly available lectins, concanavalin A (ConA) is a tetrameric protein with four binding sites for specific interaction with terminal D-mannose residues [19]. It is known to bind specifically to molecules containing $\alpha$-Dmannosyl and $\alpha$-D-glucosyl residues, this binding being reversible with the use of Methyl $\alpha$ mannopyranoside [20-22]. It has been used to characterize the presence of sugars on the surface of colloidal carriers, with the binding of the ConA to the nanoparticle surface monitored by dynamic light scattering (DLS). Mannosylated nanoprodrugs were diluted in ConA buffer (5mM HEPES, $150 \mathrm{mM} \mathrm{NaCl}, 1 \mathrm{mM} \mathrm{CaCl}_{2}, 1 \mathrm{mM} \mathrm{MnCl}$, $\mathrm{pH}$ 6.5) to the final concentration of $100 \mu \mathrm{g} / \mathrm{mL}$. After 5 minutes of size measurement at $25^{\circ} \mathrm{C}$ (Zetasizer Nano ZS), $80 \mu \mathrm{L}$ of a $100 \mu \mathrm{M}$ ConA in the abovedescribed buffer was added to the nanoparticle suspensions (ConA final concentration of $2 \mu \mathrm{M}$ ), the particle size is measured for a further 35 minutes [12]. After 40 minutes a solution of Methyl $\alpha$-Dmannopyranoside $(10 \mathrm{mM})$ was added as a desorption agent of ConA [23] and the size of the suspension was measured by DLS. 


\subsection{Cell culture}

All in vitro cell culture assays were performed on murine macrophage cell line RAW 264.7 obtained from ATCC (USA), cultured in DMEM (Dulbecco's Modified Eagle's Medium) culture medium supplemented with $10 \%$ fetal bovine serum (FBS), penicillin $\mathrm{G}(10,000 \mathrm{unit} / \mathrm{mL})$ and streptomycin $(10 \mathrm{mg} / \mathrm{mL})$, maintained in a humidified incubator at $37^{\circ} \mathrm{C}$ supplied with $5 \% \mathrm{CO}_{2}$. Cells were used between passages 5 to 15 .

\subsection{Cellular viability}

The effect of the formulations on the cellular viability was studied on the RAW 264.7 cell line using the MTT (3-(4,5-dimethylthiazolyl-2)-2,5-diphenyltetrazolium bromide) colorimetric assay. Cells were seeded in 96-well plates at the density of $1 \times 10^{4}$ cells/well ( $24 \mathrm{~h} \mathrm{Test)}$ and $2 \times 10^{3}$ cells/well ( $48 \mathrm{~h}$ Test) and incubated for $24 \mathrm{~h}$ until $80 \%$ confluence. PEGylated and mannosylated Nanoprodrugs were added at the final concentrations between $10^{-9} \mathrm{M}$ and $10^{-4} \mathrm{M}$ (eq Bud, which corresponds to a NP concentration from $1.34 .10^{-6} \mathrm{mg} / \mathrm{mL}$ up to $0.134 \mathrm{mg} / \mathrm{mL}$ ), with the corresponding concentrations being equivalent to free budesonide, solubilized in ethanol, and then diluted in the culture medium to reach the same concentration of budesonide as nanoprodrugs. After 24 hours and 48 hours of incubation, $20 \mu \mathrm{L}$ of a $5 \mathrm{mg} / \mathrm{mL}$ yellow tetrazolium MTT (3-(4,5-dimethylthiazolyl-2)-2,5diphenyltetrazolium bromide) solution was added to each well and incubated for 1 additional hour or until the formation of the purple formazan crystals, resulting from the MTT reduction by metabolically active cells. Afterward, the culture medium was replaced by $200 \mu \mathrm{L}$ of DMSO to dissolve formazan crystals and the absorbance was measured at $570 \mathrm{~nm}$. The percentage of viable cells was calculated as the absorbance ratio between nanoparticle-treated and untreated control cells.

\subsection{Cytokine release}

The anti-inflammatory effect of mannosylated nanoprodrugs was tested by inducing an inflammatory reaction in RAW 264.7 cells with the help of Escherichia coli lipopolysaccharides (LPS). For the cytokine quantification, RAW 264.7 cells were seeded in 24 -well plates at a cell density of $4 \times 10^{4}$ cells/well in culture medium and were incubated for 48 hours until $80 \%$ confluency. Then, the medium was replaced by fresh medium alone or fresh medium with LPS at $1 \mu \mathrm{g} / \mathrm{mL}$ to induce inflammation, and plates were incubated for another 3 hours. Afterward, mannosylated nanoprodrugs $75 / 25$ and free budesonide at four concentrations diluted in culture medium: $10^{-7} \mathrm{M}$, $10^{-8} \mathrm{M}, 10^{-9} \mathrm{M}$ and $10^{-10} \mathrm{M}$ (eq Bud) were added [24-27]. The culture medium alone was used as a negative control and LPS $1 \mu \mathrm{g} / \mathrm{mL}$ as a positive control. After 24,48 , and 72 hours of incubation with the treatments, cell supernatants were collected and frozen at $-20^{\circ} \mathrm{C}$ until analysis was performed. 
Cells were detached and counted. Mouse inflammatory cytokines, TNF $\alpha$ and MCP-1 were quantified using a Cytometric Beads Array (CBA) detection kit (BD Biosciences, USA). In each test tube, 50 $\mu \mathrm{L}$ of mouse inflammation capture bead suspension was added, completed with either $50 \mu \mathrm{L}$ of standards solution $(20-5000 \mathrm{pg} / \mathrm{mL})$ or $50 \mu \mathrm{L}$ of supernatants samples. Phycoerythrin (PE) detection reagent was added $50 \mu \mathrm{l}$ to each tube and incubation for $2 \mathrm{~h}$ at room temperature was performed. Samples were washed with $1 \mathrm{~mL}$ wash buffer provided in the kit and tubes were centrifuged $(200 \mathrm{~g}, 5 \mathrm{~min})$ to recover the pellet. $300 \mu \mathrm{L}$ of wash buffer was added to resuspend the pellet and samples were quantified with the BD Accuri C6 Cytometer (BD Biosciences, USA). Cytokines secretion was analyzed with the FACP Array ${ }^{\mathrm{TM}}$ Software and were obtained as $\mathrm{pg} / \mathrm{mL}$ concentrations. All measurements were performed in triplicate.

\subsection{Nanoprodrug uptake}

The internalization/uptake of nanoprodrugs in RAW 264.7 cells was analyzed by confocal laser scanning microscopy (CLSM) and quantified by Flow Cytometry, using Rhodamine labeled nanoprodrugs (labeled with $1 \%$ lissamine-rhodamine).

For the microscopic analysis, RAW 264.7 cells were seeded in culture medium in a 6-well plate containing a $0.17 \mathrm{~mm}$ diameter coverslip, at a cell density of $4 \times 10^{4}$ cells/well ( $24 \mathrm{~h}$ treatment) and 2 $\times 10^{4}$ cells/well for $48 \mathrm{~h}$ ), remaining in the incubator for $24 \mathrm{~h}$ until reaching $80 \%$ of confluence. After this period, the culture medium was replaced by a fresh medium in the control case or medium containing $25 \mu \mathrm{g} / \mathrm{mL}$ BP nanoprodrugs (PEGylated and mannosylated nanoprodrugs containing budesonide palmitate), these plates were incubated for another $24 \mathrm{~h}$ and $48 \mathrm{~h}$. Observations were performed with a LSM 510 (Zeiss - Meta) confocal microscope equipped with Helium-Neon (543 nm, $5 \mathrm{~mW}$ ) laser and a plan-apochromat $63 \mathrm{X}$ objective. Red fluorescence was observed with a long-pass $560 \mathrm{~nm}$ emission filter under $543 \mathrm{~nm}$ laser illumination. All imaging parameters were kept constant for reliable comparison between experiments.

For flow cytometry measurements, cells were seeded in 12 -well plates at the density of $4 \times 10^{4}$ cells/well and incubated for $24 \mathrm{~h}$ until $80 \%$ confluence. PEGylated and mannosylated nanoparticle formulations were added at the final concentration of $25 \mu \mathrm{g} / \mathrm{mL}$ and incubated for $1,3,5,7,24$, and 48 hours, at $4^{\circ} \mathrm{C}$ and $37^{\circ} \mathrm{C}$. Cells were then washed with PBS, centrifuged at $300 \mathrm{~g}$ at $4^{\circ} \mathrm{C}$ for 5 minutes, and resuspended in $200 \mu \mathrm{L}$ PBS $[28,29]$. Samples were analyzed using a flow cytometer (Accuri C6, BD Biosciences, USA) at the excitation and emission wavelengths of 570 and $590 \mathrm{~nm}$, respectively. For the quantification of the fluorescence intensity relative to the entry of the nanoprodrugs into the cells, the mean fluorescence intensity (MFI) was calculated by the fluorescence ratio between untreated cells (control) and treated cells. 
To verify whether the uptake mechanism of mannosylated nanoprodrugs was arising from binding to the mannose receptor, an inhibition study was performed with mannan, a known mannose receptor with 100 times more affinity to mannose receptor than mannose itself. Cells were seeded in 12-well plates at the density of $4 \times 10^{4}$ cells/well and incubated for $24 \mathrm{~h}$ until $80 \%$ confluence, cultured in DMEM culture medium supplemented with FBS and Penicillin-streptomycin. After $24 \mathrm{~h}$, the medium was replaced with a fresh medium DMEM containing $1 \%$ bovine serum albumin (BSA), 25 mM HEPES, $3 \mathrm{mM} \mathrm{CaCl}_{2}$, and mannan $1 \%$, and the cells were incubated for $2 \mathrm{~h}$. PEGylated and mannosylated nanoprodrugs were added at the final BP concentration of $25 \mu \mathrm{g} / \mathrm{mL}$ and incubated for 24 and 48 hours at $37^{\circ} \mathrm{C}$. DMEM medium was used for negative control [30-33]. Flow cytometry experiments were performed as described above.

\subsection{Statistical analysis}

Results were reported as mean \pm standard error of the mean (SEM). Statistical analysis was performed using the GraphPad Prism 7.0 software.

\section{RESULTS AND DISCUSSIONS}

\subsection{Synthesis of Budesonide Palmitate (BP) and DSPE-PEG-Man}

The synthesis of budesonide palmitate was readily accomplished, with a yield of $67 \%$ (Figure S1). The reaction was confirmed by nuclear magnetic resonance $\left({ }^{1} \mathrm{H} N M R\right)$ at $300 \mathrm{MHz}$ in deuterated chloroform $\left(\mathrm{CDCl}_{3}\right)$. The synthesis of DSPE-PEG-Man was successfully performed, with a yield between 50-75\% (Figure 1A). DSPE-PEG-Man NMR analysis exhibited two peaks at $\delta$ 7.0-7.2 (MPITC phenyl-H, arrows in Fig. 1B), indicating successful grafting of mannose on DSPE-PEG-NH $\mathrm{NH}_{2}$ (Figure $1 \mathrm{~A}$ ). However, when DSPE-PEG-Man was analyzed by HPLC coupled to UV or ELSD detectors, it was possible to detect and quantify a small amount of free DSPE-PEG- $\mathrm{NH}_{2}$ that did not react with $\alpha$-DMannopyranosylphenyl isothiocyanate (MPITC) (around 12\%, $n=8$ ). Figure S2 presents a DSPE-PEGMan chromatogram analyzed by HPLC-ELSD-UV. In red, DSPE-PEG-Man (UV detector) presents a retention time of $6.5 \mathrm{~min}$, identical to the retention time of DSPE-PEG-NH $\mathrm{N}_{2}$ in black (ELSD detector). DSPE-PEG-Man was therefore quantified by UV- detection and DSPE-mPEG and DSPE-PEG-NH $\mathrm{NH}_{2}$ by ESLD detection. 
A
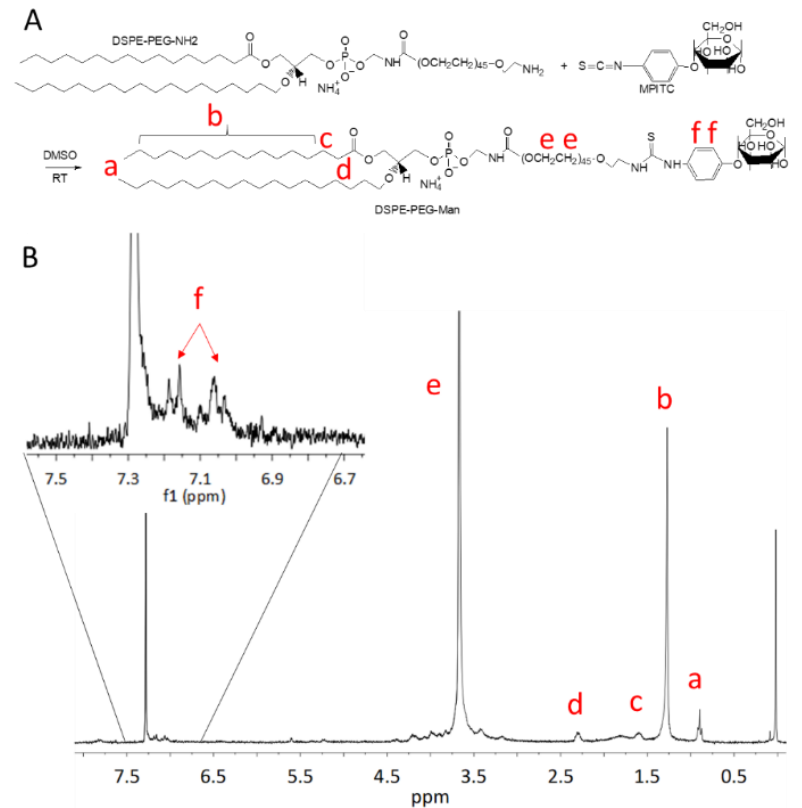

Figure 1. A) DSPE-PEG-Man synthesis and B) ${ }^{1} \mathrm{H}$ NMR spectrum of DSPE-PEG-Man.

\subsection{Nanoprodrug characterization and budesonide palmitate loading}

Nanoprodrugs were obtained using various ratios of DSPE-PEG-Man and DSPE-mPEG and prepared by emulsion-evaporation (EE). A final BP concentration of $1.25 \mathrm{mg} / \mathrm{mL}$ in water was fixed, using a 1 to 1 ratio for BP and total lipids (PEGylated and Mannosylated). Small particles of 180-200 nm were obtained, except for the formulation 100/0 (containing only DSPE-PEG-Man), which displayed a hydrodynamic diameter around $400 \mathrm{~nm}$ (Table 2). The Pdl remained below 0.2 for the ratios 0/100 to $75 / 25$, indicating rather monodisperse suspensions. Nevertheless, the Pdl of formulation with only DSPE-PEG-Man (100/0) was around 0.5 suggesting very polydisperse suspensions. This can be explained by the zeta potential values. Indeed, as the content of mannosylated lipid is increasing, the zeta potential of the nanoprodrugs increases from $-30 \mathrm{mV}$ up to almost 0 . This zeta potential increase is due to the presence of DSPE-PEG-NH $\mathrm{NH}_{2}$ that did not react with the mannose moiety as pointed out above. Since DSPE-PEG- $\mathrm{NH}_{2}$ is positively charged, as mannosylated lipid proportion increases in the formulations, the zeta potential progressively becomes neutral for these specific formulations. The neutral zeta potential leads to aggregation since nanoprodrugs are not electrostatically stabilized anymore explaining large sizes and high polydispersity.

The stability of the nanoprodrugs stored at $4^{\circ} \mathrm{C}$ was monitored for one month by measuring the size, Pdl and zeta potential (Figure S3). All formulations were stable until 30 days, except for the 100/0 formulations for the reasons explained above. Nanoprodrugs obtained with 100/0 Mannosylated lipid ratio were discarded due to their instability. 
Table 2: Initial characteristics of the formulations in terms of size, PDI and zeta potential.

\begin{tabular}{|l|c|c|c|}
\hline $\begin{array}{l}\text { Formulation } \\
\text { DSPE-PEG-Man/DSPE-mPEG }(\mathbf{w} / \mathbf{w})\end{array}$ & $\begin{array}{c}\text { Size } \\
(\mathbf{n m})\end{array}$ & PDI & $\begin{array}{c}\text { Zeta } \\
\text { Potential } \\
(\mathbf{m V})\end{array}$ \\
\hline \multicolumn{4}{|c|}{ Emulsion evaporation (EE) } \\
\hline $0 / 100$ & $168 \pm 18$ & 0.13 & $-35 \pm 4$ \\
\hline $25 / 75$ & $168 \pm 10$ & 0.11 & $-28 \pm 2$ \\
\hline $50 / 50$ & $193 \pm 3$ & 0.10 & $-22 \pm 6$ \\
\hline $75 / 25$ & $202 \pm 21$ & 0.12 & $-14 \pm 5$ \\
\hline $100 / 0$ & $398 \pm 172$ & 0.51 & $-3 \pm 8$ \\
\hline
\end{tabular}

The encapsulation efficiency and drug loading of the mannosylated nanoprodrugs were then determined. After separation by ultracentrifugation, non-encapsulated BP and DSPE-PEG-Man present in the supernatant were quantified by HPLC-UV and HPLC-ELSD and their amount in the nanoprodrugs was calculated indirectly (Figure 2A). The encapsulation efficiency of BP was ranging from $89 \%$ up to $107 \%$ with no significant differences proving that all the prodrug is associated with nanoprodrugs. Results were different for DSPE-PEG-Man encapsulation. One can observe a DSPEPEG-Man encapsulation efficiency from $14 \%$ up to $37 \%$, which increased as the DSPE-PEG-Man/DSPEmPEG ratio increased. 


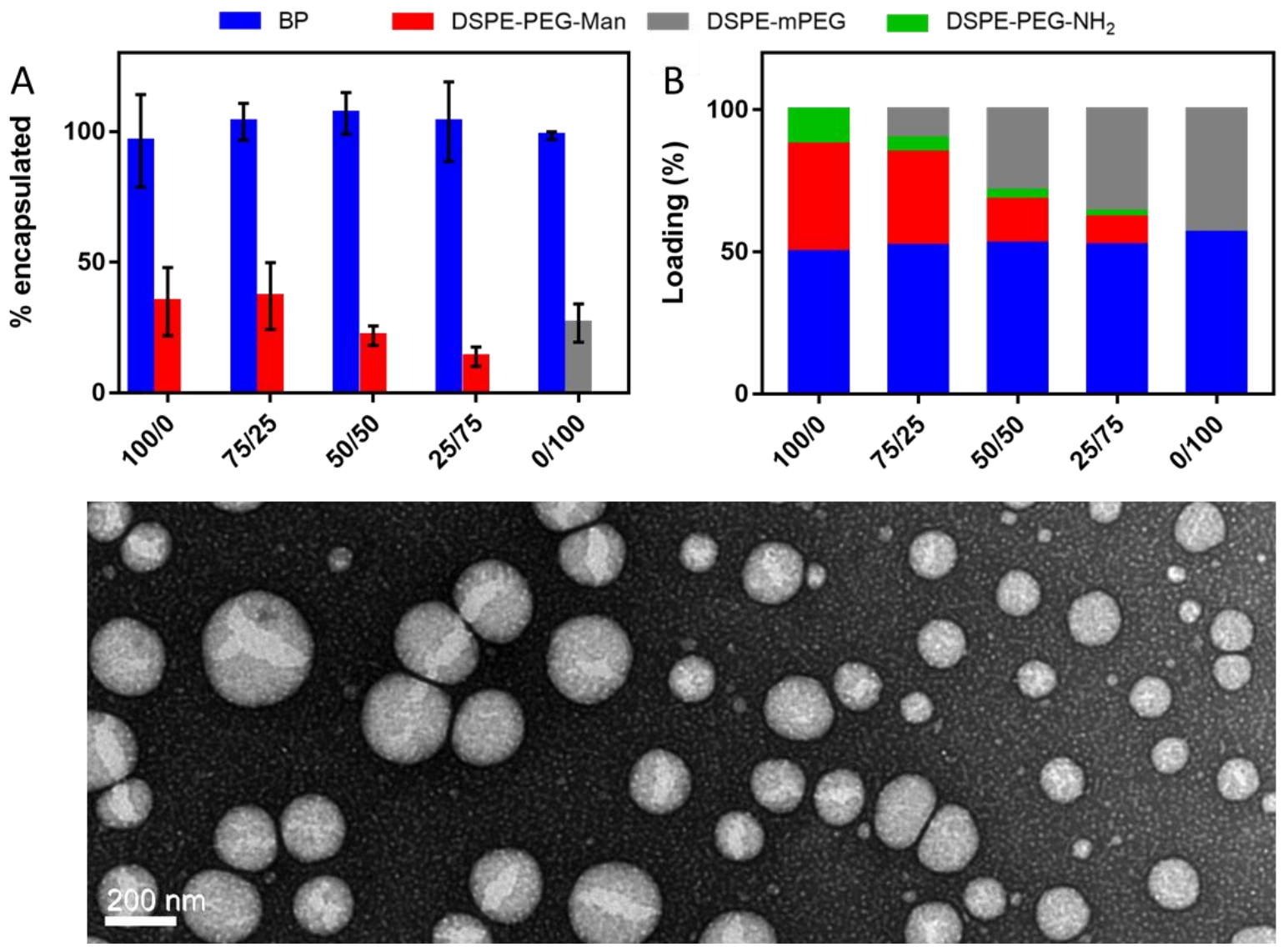

Figure 2. BP (blue), DSPE-PEG-Man (red), DSPE-mPEG (grey), and DSPE-PEG-NH2 (green) encapsulation efficiency (A) loading (B), according to the proportion of components $(n=3)$. Typical image of Budesonide nanoparticles stabilized by DSPE-mPEG (C).

Based on the amounts of encapsulated molecules, BP, DSPE-PEG-Man, DSPE-PEG-NH $\mathrm{N}_{2}$ and DSPEmPEG loadings were calculated (Figure 2B). The quantification of DSPE-PEG-NH ${ }_{2}$ and DSPE-mPEG was performed by HPLC-ELSD detection method. However, when these compounds are present in the same formulation, as ratios $75 / 25,50 / 50$, and $25 / 75$, they showed similar retention times (Figure S2), making it impossible to separate them. For this reason, we first quantified the amount of unreacted DSPE-PEG-NH $\mathrm{NH}_{2}$ present in several synthesis batches of DSPE-PEG-Man, around $12 \%$. Then, to calculate the compound loading, we used the following data: 1) quantification of DSPE-PEG-Man in each formulation (HPLC-UV); 2) theoretical total lipids in each formulation (1.25 mg/mL); 3) lipid ratio in each formulation; 4) the difference between theoretical total lipids and quantified DSPE-PEG-Man = other lipids; 5) quantity obtained as other lipids = DSPE-mPEG and/or DSPE-PEG-NH ${ }_{2}$, which we calculated considering the ratio used and DSPE-PEG-NH$H_{2}$ as $12 \%$ of dosed DSPE-PEG-Man. Formulations 100/0 (100\% DSPE-PEG-Man) and 0/100 (100\% DSPE-mPEG) (Figure 2B), were calculated by direct dosing of DSPE-PEG-NH ${ }_{2}$ and DSPE-mPEG, respectively, since these were the only other lipids present in these formulations. 
Nanoprodrugs presented a BP loading from 48 to $60 \%(\mathrm{w} / \mathrm{w})$. This high prodrug loading is similar to what was obtained for dexamethasone palmitate loading using the same processes $[10,11]$. We also observe that the higher the initial DSPE-PEG-Man, the higher the DSPE-PEG-Man loading which varied from 10 to $45 \%$ by weight. Nanoparticle morphology displays spherical shape which distribution correlates with particle size distribution (Figure $2 \mathrm{C}$ ).

\subsection{Lectin Agglutination Test}

To verify the presence and biological activity of mannose present on the nanoprodrug surface, the lectin agglutination test was used (Figure 3). On one hand, control obtained using PEGylated nanoprodrugs $(0 / 100)$ and amine-terminated nanoprodrugs $\left(\mathrm{NH}_{2}\right)$ did not show any change regarding their size distribution in the presence of ConA, indicating no interactions with the protein, for both formulation processes (Figure 3). On the other hand, mannosylated nanoprodrugs exhibited a size increase when ConA was added (Figure 3). The higher the mannosylated lipid content, the stronger the size increase. This confirms that the extent of agglomeration depends on the amount of mannose present on nanoprodrug surface. When methyl $\alpha$-D-mannopyranoside was added in excess to compete with mannosylated nanoprodrugs, aggregation was reversed, and the initial nanoparticle size was recovered as ConA desorbed from nanoparticle surface and interacted with methyl $\alpha$-Dmannopyranoside (Figure 3).

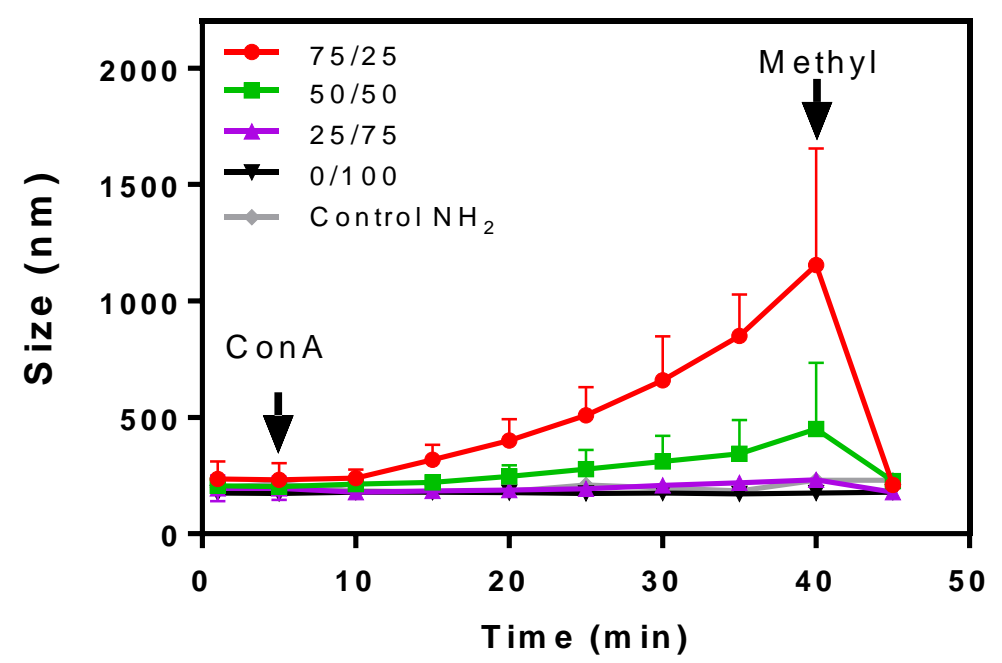

Figure 3. Reaction of mannosylated nanoprodrugs to the addition of $100 \mu \mathrm{M}$ ConA (time $=5 \mathrm{~min}$ ) and $10 \mathrm{mM}$ Methyl $\alpha$-D-mannopyranoside (time $=40 \mathrm{~min})(\mathrm{n}=3)$.

For the following experiments, as nanoprodrugs prepared with the highest amount of mannose were more prone to agglutination when lectin was added, the 75/25 formulation was chosen for further experiments and compared with plain PEGylated nanoparticles (0/100). 


\subsection{Cellular viability}

Before conducting targeting experiments, the potential toxicity of mannosylated nanoprodrugs was evaluated on RAW 264.7 cells using MTT assay. According to ISO guideline for MTT assay, potential cytotoxicity is considered when cell viability decreases below $70 \%$ of the control [34]. A range of concentrations from $10^{-9} \mathrm{M}$ to $10^{-4} \mathrm{M}$ (equivalent budesonide, which corresponds to a NP concentration from $1.34 .10^{-6} \mathrm{mg} / \mathrm{mL}$ up to $0.134 \mathrm{mg} / \mathrm{mL}$ ) was tested and compared to controls: budesonide and PEGylated nanoprodrugs. After 24 hours incubation, mannosylated and PEGylated nanoprodrugs clearly showed no cytotoxicity up to $3 \times 10^{-5} \mathrm{M}$ (eq budesonide, which corresponds to a NP concentration of $0.04 \mathrm{mg} / \mathrm{mL}$ ), while free budesonide showed no cytotoxicity up to $7 \times 10^{-5} \mathrm{M}$ (Figure 4A). After 48 hours of incubation, mannosylated nanoprodrugs clearly showed no cytotoxicity up to $2 \times 10^{-5} \mathrm{M}$ (eq budesonide, which corresponds to a NP concentration of $0.026 \mathrm{mg} / \mathrm{mL}$ ), while free budesonide and PEGylated nanoprodrugs showed no cytotoxicity up to $3 \times 10^{-5} \mathrm{M}$ (Figure 4B). One cannot see any significant differences between PEGylated and mannosylated formulation nor free budesonide. Our results are in agreement with previous data demonstrating a relative safety of budesonide versus macrophages up to a concentration in the micromolar range [35].

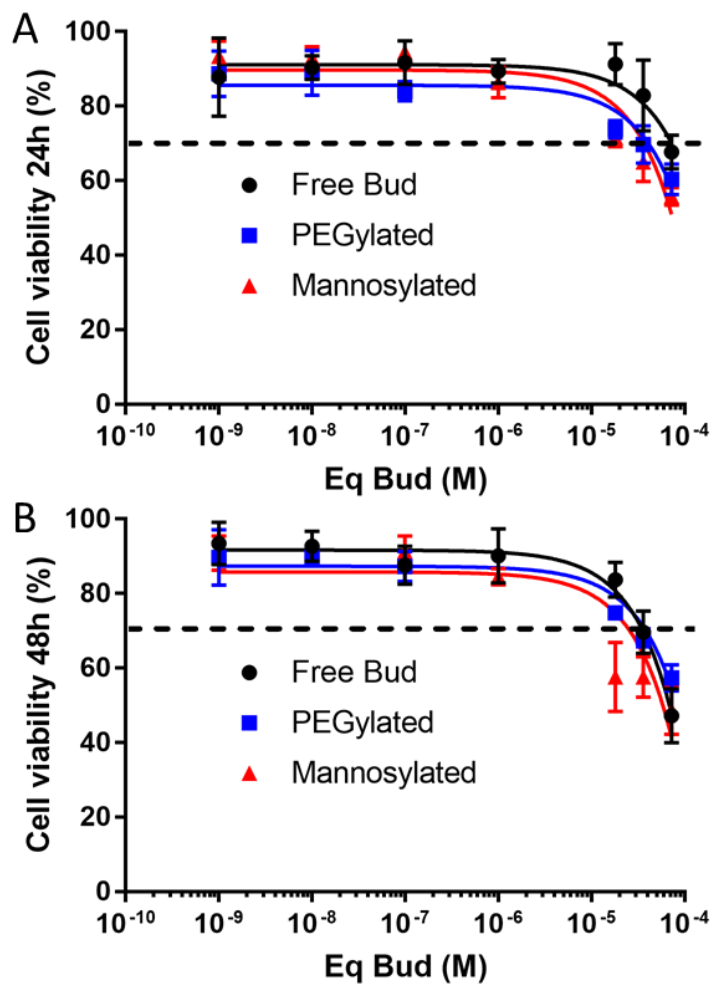

Figure 4. Cell viability of the RAW 264.7 cell line incubated with either free budesonide or PEGylated and mannosylated nanoprodrugs at different equivalent concentrations of budesonide, after $24 \mathrm{~h}(\mathrm{~A})$ and $48 \mathrm{~h}$ incubation (B) (data are presented as mean $\pm S D, n=3$ ). 


\subsection{Nanoprodrug cellular uptake}

Nanoprodrug internalization in the RAW 264.7 cell line was studied using flow cytometry using nanoprodrugs labeled with a $1 \%$ lissamine-rhodamine. Tests were performed comparing mannosylated nanoprodrugs (75/25), PEGylated nanoparticles (0/100), and control (non-treated cells). Figure $5 \mathrm{~A}$ shows that the internalization of nanoprodrugs either PEGylated or mannosylated can be already detected after 3 hours. After 24 hours of incubation, a significantly greater internalization of mannosylated nanoprodrugs as compared to PEGylated nanoprodrugs was observed. This finding holds also for $48 \mathrm{~h}$ incubation. Mannosylated nanoprodrugs presented an uptake significantly higher than PEGylated nanoprodrugs. To evaluate if nanoparticle uptake was energy-dependent, uptake was evaluated at $4{ }^{\circ} \mathrm{C}$ (Figure 5B). Results show no uptake at this temperature for either PEGylated or mannosylated nanoprodrugs confirming that the uptake observed at $37^{\circ} \mathrm{C}$ occurs by an energy-dependent uptake mechanism.

To confirm a mannose receptor-mediated uptake, a mannan inhibition study was performed, since mannan is a known ligand of the mannose receptor. RAW 264.7 cells were either incubated with mannan at $1 \mathrm{mg} / \mathrm{mL}$ or just cell culture medium and then treated with PEGylated or mannosylated nanoprodrugs (Figure 5C). After 24 hours of incubation, PEGylated nanoprodrugs did not show significant differences when the cells were incubated with mannan, while a statistically significant decrease in cellular uptake was observed in the case of mannosylated nanoprodrugs. Similar results were found after 48 hours of incubation. The results suggest that the pre-incubation of cells with mannan probably leads to the saturation of mannose receptors on the cell surface, confirming mannose receptor-mediated uptake of mannosylated nanoprodrugs. Our results are in agreement with mannose receptor-mediated mechanisms as shown by others [33].

Confocal laser scanning microscopy (CLSM) experiments were carried out to confirm the internalization of mannosylated and PEGylated nanoprodrugs (Figure $5 \mathrm{D}$ and E). Both PEGylated and mannosylated nanoprodrugs were found in the cytoplasm of macrophages. After 48 hours of incubation, it was possible to identify an enhanced uptake for mannosylated nanoprodrugs (Figure 5E), when compared to PEGylated nanoprodrugs (Figure 5D). These findings confirm the results obtained by flow cytometry.

In conclusion, these studies provide evidence that mannosylated nanoprodrugs can be used to target mannose receptors present on macrophage surface. Mannosylated nanoprodrugs enter macrophages by receptor-mediated endocytosis via surface-bound mannose receptors. These results are in line with previous studies showing enhanced cellular uptake of mannose-conjugated nanoparticulate systems on several macrophages cell lines [36-39]. 

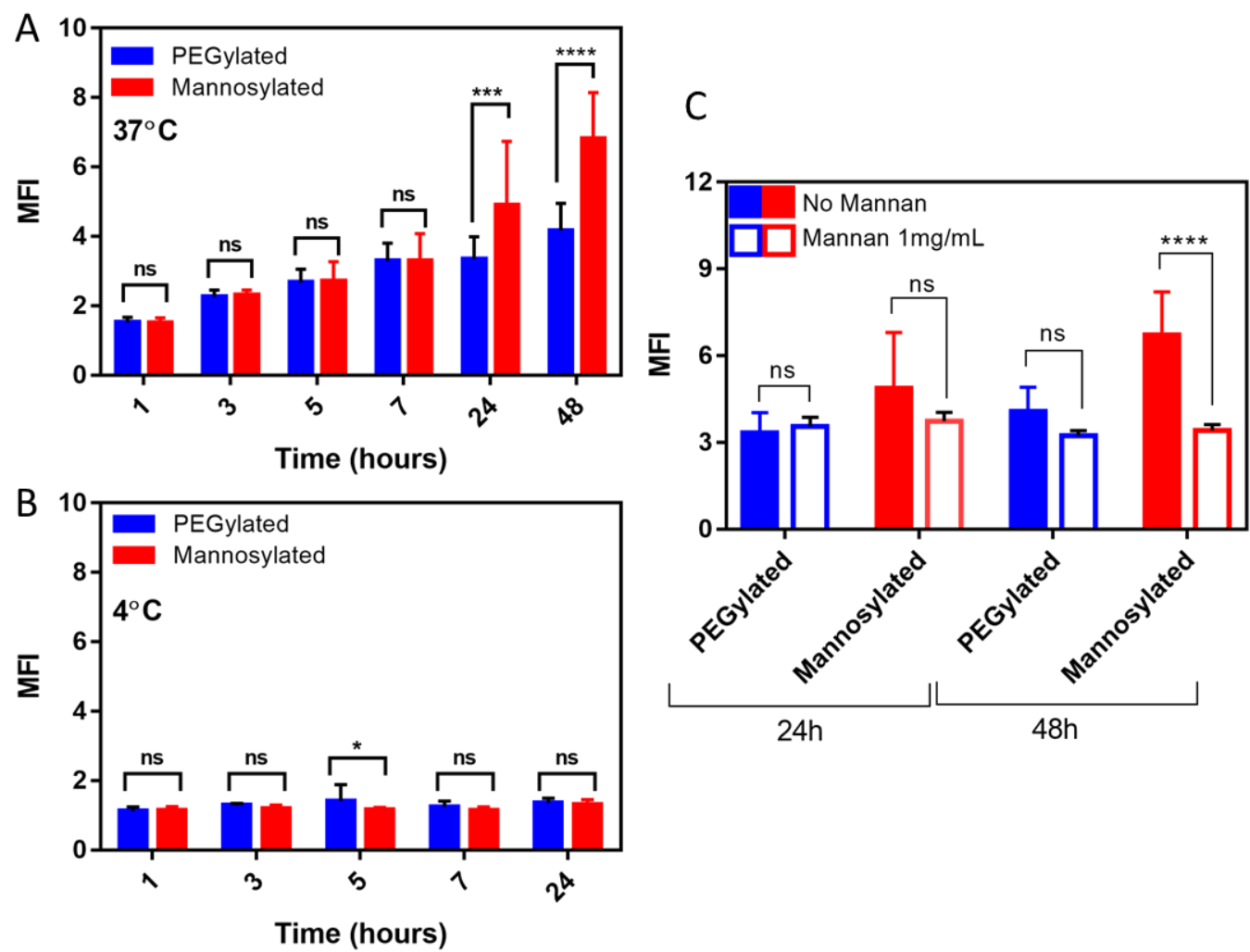

D PEGylated nanoprodrugs

\section{E Mannosylated nanoprodrugs}
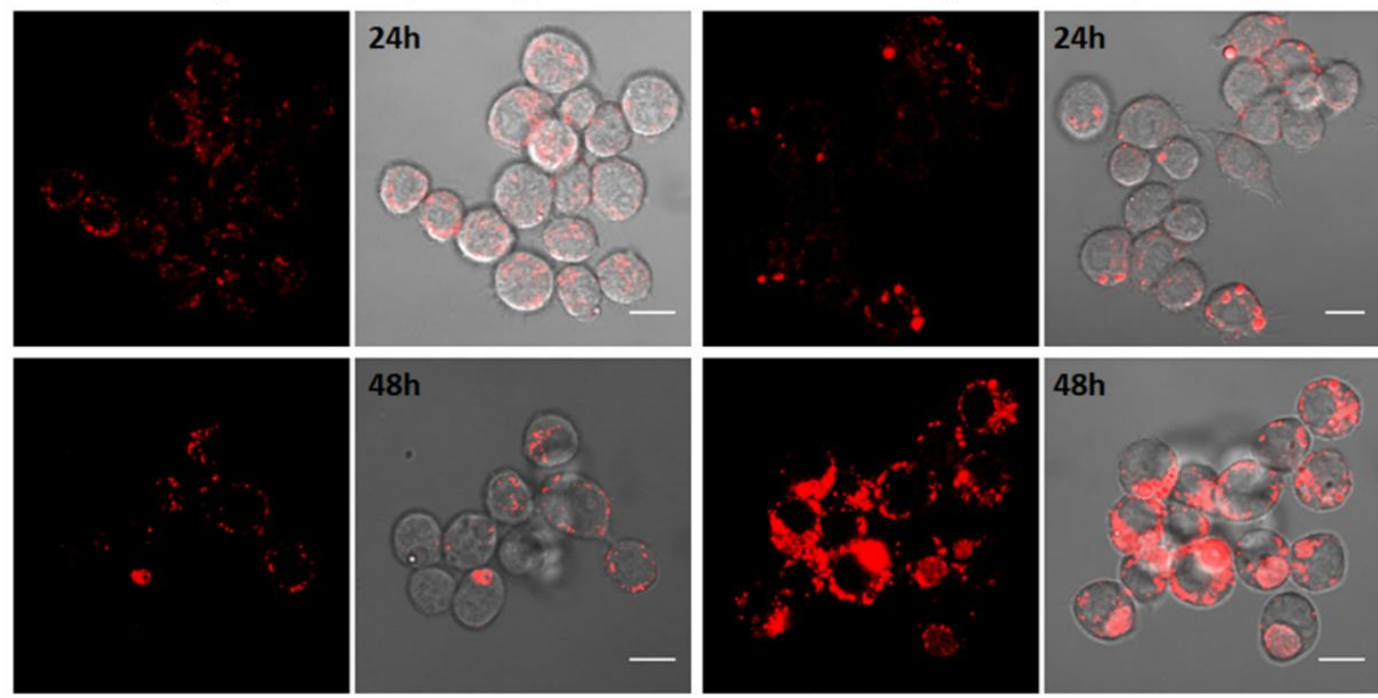

Figure 5. A) Uptake kinetics of PEGylated nanoprodrugs $(0 / 100$, blue) and mannosylated nanoprodrugs $\left(75 / 25\right.$, red) by RAW 264.7 cells after $1,3,5,7,24$ and $48 \mathrm{~h}$ incubation at $37{ }^{\circ} \mathrm{C}$, presented as MFI compared to untreated cells. B) Uptake kinetics uptake after 1, 3, 5, 7 and $24 \mathrm{~h}$ incubation at $4^{\circ} \mathrm{C}$, presented as MFI compared to untreated. C) Uptake after 24 and $48 \mathrm{~h}$ incubation at $37^{\circ} \mathrm{C}$, in competition or not with $1 \mathrm{mg} / \mathrm{mL}$ mannan, presented as MFI compared to untreated. Statistical analysis was performed with two-way ANOVA followed by Tukey's multiple comparisons test $(n=9)$. ns: not significant, ${ }^{*} p<0.05,{ }^{* *} p<0.01,{ }^{* * *} p<0.001,{ }^{* * * *} p<0.0001$. Confocal Microscopy to evaluate the uptake of (D) PEGylated nanoprodrugs and (E) mannosylated nanoprodrugs after $24 \mathrm{~h}$ and $48 \mathrm{~h}$ incubation. Scale bar $=10 \mu \mathrm{m}$. 


\subsection{Cytokine release}

The anti-inflammatory activity of mannosylated nanoprodrugs was evaluated by flow cytometry. The release of pro-inflammatory cytokines (MCP-1 and TNFa) by LPS-activated macrophages RAW 264.7 was quantified after their incubation for 48 hours with PEGylated, mannosylated nanoprodrugs or free budesonide (Figure 6). Low concentrations of BP (eq budesonide) from $10^{-10} \mathrm{M}$ to $10^{-7} \mathrm{M}$ were chosen as therapeutically relevant concentrations $[27,40]$. For MCP-1, a significant decrease was observed for both treatments. Concerning TNFa, mannosylated nanoprodrugs showed a strong and significant reduction with no dose-effect, observed, meaning that the lower concentration of nanoprodrugs $\left(10^{-10} \mathrm{M}\right)$ was sufficient to reach the expected anti-inflammatory effect. Taken all together, these data demonstrate that mannosylation and the use of budesonide palmitate are consistent with drug release from the prodrug and further anti-inflammatory activity of budesonide.
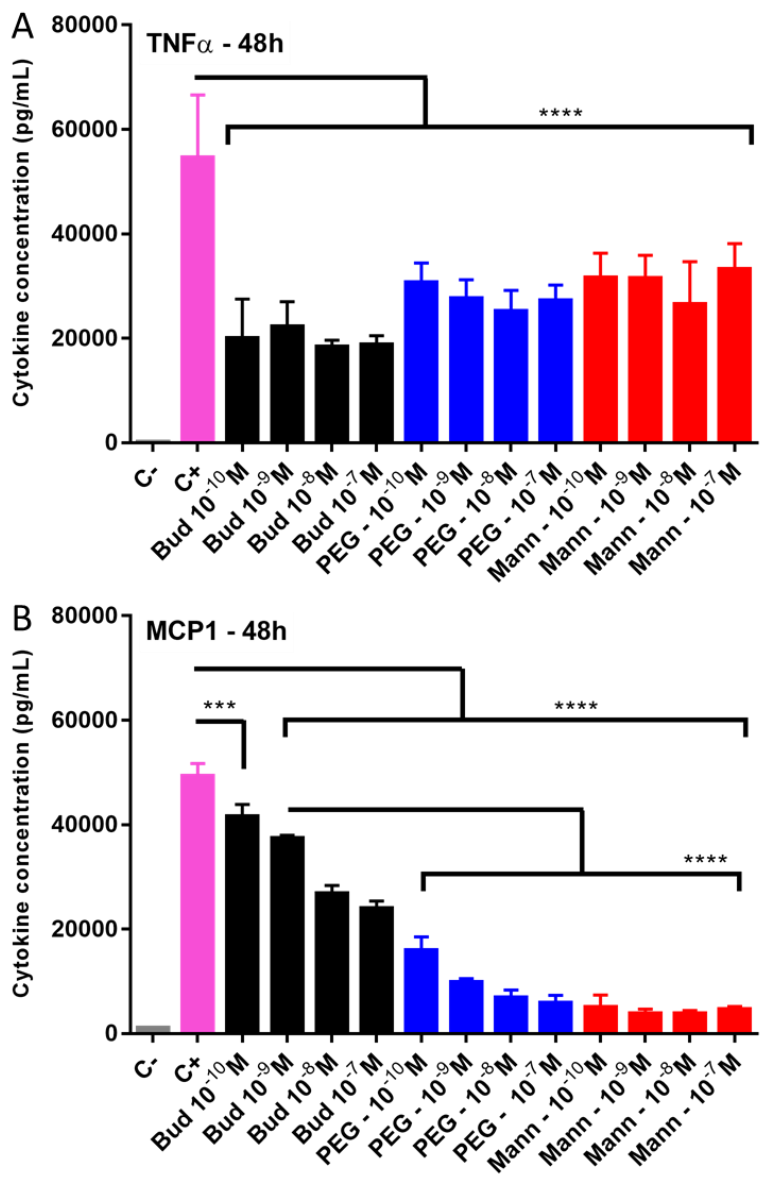

Figure 6. Quantification of cytokine TNF- $\alpha$ (A) and MCP-1 (B) release from RAW 264.7 macrophages without activation by LPS (C-, grey), with induction by LPS $1 \mu \mathrm{g} / \mathrm{mL}(\mathrm{C}+$, pink), $48 \mathrm{~h}$ after treatment with Budesonide (black), PEGylated nanoprodrugs (blue) and mannosylated nanoprodrugs (red) as a function of equivalent budesonide concentration. Results are represented as mean \pm SD $(n=3)$. Statistical analysis was performed with a one-factor ANOVA followed by a Tukey post-hoc test. ns = not significant, $* * * \mathrm{p}<0.001, * * * \mathrm{p}<0.0001$. 


\section{Conclusions}

In this study, we have functionalized budesonide palmitate nanoprodrugs with mannose to improve macrophage targeting and drug uptake. A lipophilic derivative of budesonide, budesonide palmitate (BP), and a mannosylated lipid, DSPE-PEG-Man were synthesized. Nanoprodrugs were formulated at different ratios of lipids DSPE-PEG-Man/DSPE-mPEG (100/0, 75/25, 50/50, 25/75) and compared to PEGylated nanoprodrugs (ratio 0/100). Mannosylated nanoprodrugs prepared with a ratio 100/0 were discarded as they presented undesired instability characteristics. All other formulations resulted in stable and homogeneous nanoprodrugs with an excellent encapsulation efficiency and BP loading (> 48\%). The biological activity of mannose ligands on nanoprodrug surface was confirmed by lectin agglutination test. In vitro studies demonstrated the safety of mannosylated nanoprodrugs, as well as the nanoparticle uptake characteristics, showing that surface mannosylation may be an interesting way to target macrophages and improve drug selectivity. Cytokine release study confirmed their anti-inflammatory activity even at very low concentrations.

\section{Declaration of competing interest}

The authors declare that they have no known competing financial interests or personal relationships that could have appeared to influence the work reported in this paper.

\section{Acknowledgments}

Ludmila Pinheiro do Nascimento was supported by a Ph.D. scholarship from CNPq - National Council of Scientific and Technologic Development (Brazil) \#233180/2014-1. The present work has benefited from the core facilities of Imagerie-Gif, (http://www.i2bc.paris-saclay.fr), member of IBiSA (http://www.ibisa.net), supported by "France-Biolmaging" (ANR-10-INBS-04-01), and the Labex "Saclay Plant Science" (ANR-11-IDEX-0003-02) with the precious help of C. Boulogne and C. Gillet. The authors would like to acknowledge the financial support provided by COST-European Cooperation in Science and Technology, to the COST Action MP1404: Simulation and pharmaceutical technologies for advanced patient-tailored inhaled medicines (Siminhale). The authors thank S. Denis for cell culture and V. Nicolas (MIPSIT, SFR-UMS-IPSIT, Université Paris-Saclay) for confocal microscopy.

\section{REFERENCES}

[1] A.K. McDonough, J.R. Curtis, K.G. Saag, The epidemiology of glucocorticoid-associated adverse events., Curr. Opin. Rheumatol. 20 (2008) 131-137.

[2] K.G. Saag, R. Koehnke, J.R. Caldwell, R. Brasington, L.F. Burmeister, B. Zimmerman, J. a Kohler, D.E. Furst, Low dose long-term corticosteroid therapy in rheumatoid arthritis: an analysis of serious adverse events., Am. J. Med. 96 (1994) 115-23.

[3] C.M. Spies, C. Strehl, M.C. van der Goes, J.W.J.J. Bijlsma, F. Buttgereit, Glucocorticoids, Best 
Pract. Res. Clin. Rheumatol. 25 (2011) 891-900. doi:10.1016/j.berh.2011.11.002.

[4] T.M. Allen, The use of glycolipids and hydrophilic polymers in avoiding rapid uptake of liposomes by the mononuclear phagocyte system, Adv. Drug Deliv. Rev. 13 (1994) 285-309. doi:10.1016/0169-409X(94)90016-7.

[5] J.M. Metselaar, M.H.M. Wauben, J.P.A. Wagenaar-Hilbers, O.C. Boerman, G. Storm, Complete remission of experimental arthritis by joint targeting of glucocorticoids with long-circulating liposomes, Arthritis Rheum. 48 (2003) 2059-2066. doi:10.1002/art.11140.

[6] C. Gómez-Gaete, N. Tsapis, M. Besnard, A. Bochot, E. Fattal, Encapsulation of dexamethasone into biodegradable polymeric nanoparticles, Int. J. Pharm. 331 (2007) 153-159. doi:10.1016/j.ijpharm.2006.11.028.

[7] C. Gómez-Gaete, E. Fattal, L. Silva, M. Besnard, N. Tsapis, Dexamethasone acetate encapsulation into Trojan particles, J. Control. Release. 128 (2008) 41-49. doi:10.1016/j.jconrel.2008.02.008.

[8] T.M. Allen, P.R. Cullis, Liposomal drug delivery systems: From concept to clinical applications, Adv. Drug Deliv. Rev. 65 (2013) 36-48. doi:10.1016/j.addr.2012.09.037.

[9] T.M. Allen, L.G. Cleland, Serum-induced leakage of liposome contents, BBA - Biomembr. 597 (1980) 418-426. doi:10.1016/0005-2736(80)90118-2.

[10] M. Lorscheider, N. Tsapis, M. ur-Rehman, F. Gaudin, I. Stolfa, S. Abreu, S. Mura, P. Chaminade, M. Espeli, E. Fattal, Dexamethasone palmitate nanoparticles: An efficient treatment for rheumatoid arthritis, J. Control. Release. 296 (2019) 179-189. doi:10.1016/j.jconrel.2019.01.015.

[11] M. Lorscheider, N. Tsapis, R. Simón-Vázquez, N. Guiblin, N. Ghermani, F. Reynaud, R. Canioni, S. Abreu, P. Chaminade, E. Fattal, Nanoscale Lipophilic Prodrugs of Dexamethasone with Enhanced Pharmacokinetics, Mol. Pharm. 16 (2019) 2999-3010. doi:10.1021/acs.molpharmaceut.9b00237.

[12] C.A. Ruge, H. Hillaireau, N. Grabowski, M. Beck-Broichsitter, O. Cañadas, N. Tsapis, C. Casals, J. Nicolas, E. Fattal, Pulmonary Surfactant Protein A-Mediated Enrichment of Surface-Decorated Polymeric Nanoparticles in Alveolar Macrophages, Mol. Pharm. 13 (2016) 4168-4178. doi:10.1021/acs.molpharmaceut.6b00773.

[13] W. Wijagkanalan, S. Kawakami, M. Takenaga, R. Igarashi, F. Yamashita, M. Hashida, Efficient targeting to alveolar macrophages by intratracheal administration of mannosylated liposomes in rats, J. Control. Release. 125 (2008) 121-130. doi:10.1016/j.jconrel.2007.10.011.

[14] C.A. Ruge, H. Hillaireau, N. Grabowski, M. Beck-Broichsitter, O. Cañadas, N. Tsapis, C. Casals, J. Nicolas, E. Fattal, Pulmonary Surfactant Protein A-Mediated Enrichment of Surface-Decorated Polymeric Nanoparticles in Alveolar Macrophages, Mol. Pharm. 13 (2016). doi:10.1021/acs.molpharmaceut.6b00773.

[15] G. Barratt, J.P. Tenu, A. Yapo, J.F. Petit, Preparation and characterisation of liposomes containing mannosylated phospholipids capable of targetting drugs to macrophages, BBA Biomembr. 862 (1986) 153-164. doi:10.1016/0005-2736(86)90479-7.

[16] D. Chen, Targeted siRNA Delivery Methods for RNAi- Based Therapies, Electron. Thesis Diss. Repos. Pap. 1245. (2013). http://ir.lib.uwo.ca/etd.

[17] N. Kim, D. Jiang, A.M. Jacobi, K.A. Lennox, S.D. Rose, M.A. Behlke, A.K. Salem, Synthesis and characterization of mannosylated pegylated polyethylenimine as a carrier for siRNA, Int. J. Pharm. 427 (2012) 123-133. doi:10.1016/j.ijpharm.2011.08.014.

[18] C. Wang, P. Liu, Y. Zhuang, P. Li, B. Jiang, H. Pan, L. Liu, L. Cai, Y. Ma, Lymphatic-targeted cationic liposomes: A robust vaccine adjuvant for promoting long-term immunological memory, Vaccine. 32 (2014) 5475-5483. doi:10.1016/j.vaccine.2014.07.081.

[19] C.D. Muller, F. Schuber, Neo-mannosylated liposomes: Synthesis and interaction with mouse Kupffer cells and resident peritoneal macrophages, BBA - Biomembr. 986 (1989) 97-105. doi:10.1016/0005-2736(89)90277-0.

[20] B.B. Agrawal, I.J. Goldstein, Specific binding of concanavalin A to cross-linked dextran gels., Biochem. J. 96 (1965) 23c-25c. doi:10.1042/bj0960023C. 
[21] B. Fontaniella, A.M. Millanes, C. Vicente, M.E. Legaz, Concanavalin A binds to a mannosecontaining ligand in the cell wall of some lichen phycobionts, Plant Physiol. Biochem. 42 (2004) 773-779. doi:10.1016/j.plaphy.2004.09.003.

[22] H. Kitano, Y. Ishino, K. Yabe, Dehydration effect on the recognition of amphiphiles with many pendent mannose residues by Concanavalin A, Langmuir. 17 (2001) 2312-2316. doi:10.1021/la000910u.

[23] L.C. Pan, C.C. Chien, A novel application of thermo-responsive polymer to affinity precipitation of polysaccharide, J. Biochem. Biophys. Methods. 55 (2003) 87-94. doi:10.1016/S0165022X(02)00180-X.

[24] S. Foillard, J. Russier, C. Seifert, H. Dumortier, E. Doris, Carbon nanotube-mediated delivery of budesonide to macrophages, RSC Adv. 6 (2016) 53282-53287. doi:10.1039/c6ra09809f.

[25] K. Kis, L. Bodai, H. Polyanka, K. Eder, A. Pivarcsi, E. Duda, G. Soos, Z. Bata-Csorgo, L. Kemeny, Budesonide, but not tacrolimus, affects the immune functions of normal human keratinocytes, Int. Immunopharmacol. 6 (2006) 358-368. doi:10.1016/j.intimp.2005.08.022.

[26] X. Wang, A. Nelson, Z.M. Weiler, A. Patil, T. Sato, N. Kanaji, M. Nakanishi, J. Michalski, M. Farid, H. Basma, T.D. LeVan, A. Miller-Larsson, E. Wieslander, K.C. Muller, O. Holz, H.

Magnussen, K.F. Rabe, X. Liu, S.I. Rennard, Anti-inflammatory effects of budesonide in human lung fibroblasts are independent of histone deacetylase 2, J. Inflamm. Res. 6 (2013) 109-119. doi:10.2147/JIR.S43736.

[27] F.M. Spoelstra, D.S. Postma, H. Hovenga, J.A. Noordhoek, H.F. Kauffman, Additive antiinflammatory effect of formoterol and budesonide on human lung fibroblasts, Thorax. 57 (2002) 237-241. doi:10.1136/thorax.57.3.237.

[28] M. Agnoletti, A. Bohr, K. Thanki, F. Wan, X. Zeng, J.P. Boetker, M. Yang, C. Foged, Inhalable siRNA-loaded nano-embedded microparticles engineered using microfluidics and spray drying, Eur. J. Pharm. Biopharm. 120 (2017) 9-21. doi:10.1016/j.ejpb.2017.08.001.

[29] X. Zeng, R. Morgenstern, A.M. Nyström, Nanoparticle-directed sub-cellular localization of doxorubicin and the sensitization breast cancer cells by circumventing GST-Mediated drug resistance, Biomaterials. 35 (2014) 1227-1239. doi:10.1016/j.biomaterials.2013.10.042.

[30] G. Szolnoky, Z. Bata-Csörgö, A.S. Kenderessy, M. Kiss, A. Pivarcsi, Z. Novák, K.N. Newman, G. Michel, T. Ruzicka, L. Maródi, A. Dobozy, L. Kemény, A mannose-binding receptor is expressed on human keratinocytes and mediates killing of Candida albicans, J. Invest. Dermatol. 117 (2001) 205-213. doi:10.1046/j.1523-1747.2001.14071.x.

[31] W. Yeeprae, S. Kawakami, F. Yamashita, M. Hashida, Effect of mannose density on mannose receptor-mediated cellular uptake of mannosylated O/W emulsions by macrophages, J. Control. Release. 114 (2006) 193-201. doi:10.1016/j.jconrel.2006.04.010.

[32] H. He, Q. Yuan, J. Bie, R.L. Wallace, P.J. Yannie, J. Wang, M.G. Lancina, O.Y. Zolotarskaya, W. Korzun, H. Yang, S. Ghosh, Development of mannose functionalized dendrimeric nanoparticles for targeted delivery to macrophages: use of this platform to modulate atherosclerosis, Transl. Res. 193 (2018) 13-30. doi:10.1016/j.trsl.2017.10.008.

[33] B. Sedaghat, R.J. Stephenson, A.K. Giddam, S. Eskandari, S.H. Apte, D.J. Pattinson, D.L. Doolan, I. Toth, Synthesis of Mannosylated Lipopeptides with Receptor Targeting Properties, Bioconjug. Chem. 27 (2016) 533-548. doi:10.1021/acs.bioconjchem.5b00547.

[34] International Organization for Standardization, Tests for in vitro cytotoxicity (ISO 109935:2009), 2009.

[35] A. Zetterlund, P.H. Larsson, C. Müller-Suur, L. Palmberg, K. Larsson, Budesonide but not terbutaline decreases phagocytosis in alveolar macrophages, Respir. Med. 92 (1998) 162-166. doi:10.1016/S0954-6111(98)90089-0.

[36] G. Shilakari Asthana, A. Asthana, D.V. Kohli, S.P. Vyas, Mannosylated chitosan nanoparticles for delivery of antisense oligonucleotides for macrophage targeting, Biomed Res. Int. 2014 (2014) 1-17. doi:10.1155/2014/526391.

[37] H.L. Jiang, M.L. Kang, J.S. Quan, S.G. Kang, T. Akaike, H.S. Yoo, C.S. Cho, The potential of mannosylated chitosan microspheres to target macrophage mannose receptors in an 
adjuvant-delivery system for intranasal immunization, Biomaterials. 29 (2008) 1931-1939. doi:10.1016/j.biomaterials.2007.12.025.

[38] P. Chaubey, B. Mishra, Mannose-conjugated chitosan nanoparticles loaded with rifampicin for the treatment of visceral leishmaniasis, Carbohydr. Polym. 101 (2014) 1101-1108. doi:10.1016/j.carbpol.2013.10.044.

[39] V. Viswanathan, H. Mehta, R. Pharande, A. Bannalikar, P. Gupta, U. Gupta, A. Mukne, Mannosylated gelatin nanoparticles of licorice for use in tuberculosis: Formulation, in vitro evaluation, in vitro cell uptake, in vivo pharmacokinetics and in vivo anti-tubercular efficacy, J. Drug Deliv. Sci. Technol. 45 (2018) 255-263. doi:10.1016/j.jddst.2018.01.017.

[40] J.M.M. Van Den Bosch, C.J.J. Westermann, J. Aumann, S. Edsbácker, M. Tönnesson, O. Selroos, Relationship between lung tissue and blood plasma concentrations of inhaled budesonide, Biopharm. Drug Dispos. 14 (1993) 455-459. doi:10.1002/bdd.2510140511. 